\title{
Animal Mimicry as OBT - Wilderness Therapy
}

Dr Bheemaiah, Anil Kumar, A.B Seattle W.A 98125

miyawaki@yopmail.com

\begin{abstract}
:
Animal Mimicry using the Alexa Skill 'Animal Sounds' for mimicking animal cries, in ritualistic group outdoor therapy, is a part of adventure, wilderness and OBT. Part of therapeutic camping. In this paper, the author conducts ten ten minute sessions of animal mimicry, channeling vital energies in a close primordial connection to the elements, twilight and nature, with a positive outcome in decreasing stress, improving attention, removing negative trauma, and improving sensori-motor coordination as indicated in a social responsiveness scale.
\end{abstract}

Keywords:

Animal Mimicry, Adventure Therapy, Wilderness Therapy, OBT, Autism Spectrum, Struggling Youth, Detox, Vitality

\section{Introduction.}

Outdoor Behavioural Therapy, includes many therapeutic exercises that connect oneself to the primal side of the subconscious being, drawing energy from this energy source and source of identity, while the animal instinct and a rich repertoire of animal language and culture, releases pent up energy, releases trauma, and provides much of the same benefits as a range of outdoor behavioural activities, from tree climbing to normal hiking and camping activities.

The Alexa Skill 'Animal Sounds', provides a set of animal sounds to mimic, that a listener can choose from, and mimic, for a primordial release of energy, a connection to one's primitive instincts and survival strategies, so essential, in an Urban setting.

\section{Background.}

OBT Therapy(Demille, 2014)(Russell \& Gass, 2014)(Gass et al., 2020; Russell \& Gass, 2014; White, 2015) is coupled with adventure therapy, usually prescribed in the context of increased stress at school or workplace, for disturbed and struggling youth and as a detox regime, with a high level of success, in double blind studies. The relationship to autism spectrum, (Byström 
et al., 2019) introduces a concept of vitality, from energies of nature and interaction with animals, which include photography, observation and mimicry, proving a vitality theory of mimicry gains as OBT. The results mentioned in this paper, consist of three key categories; reduce stress and instill calm, arouse curiosity and interest, and attract attention spontaneously.

(Website, n.d.)Ditza A Zachor et al. prove, in a study on 51 subjects over 13 weeks , an improvement on a social responsive scale, leading to speculations that experiential therapies in OBT, can lead with primordiality rechanneling to better cognitive, social and coordinative skills of sensory-motor machinery, leading to lower stress and better social responsiveness. ([No title], n.d.)M.J Hoag, describes a study on 297 individuals finding significant change on measures of life effectiveness, motivation for therapy, therapeutic alliance, and dysfunctional attitudes.

\section{Methods.}

The author conducted a series of animal mimicry experiments, using the above mentioned Alexa Skill, for a duration of 10 to fifteen minutes in a wilderness setting, close to Swede Hollow Park, at St Paul, in Minneapolis, USA. Therapeutic benefits were observed personally, with respect to social skills, leadership qualities, relationships to humans and animals and in reduction of trauma, anxiety and panic attacks.
The particular utility with respect to OBT for the autism spectrum is emphasized.
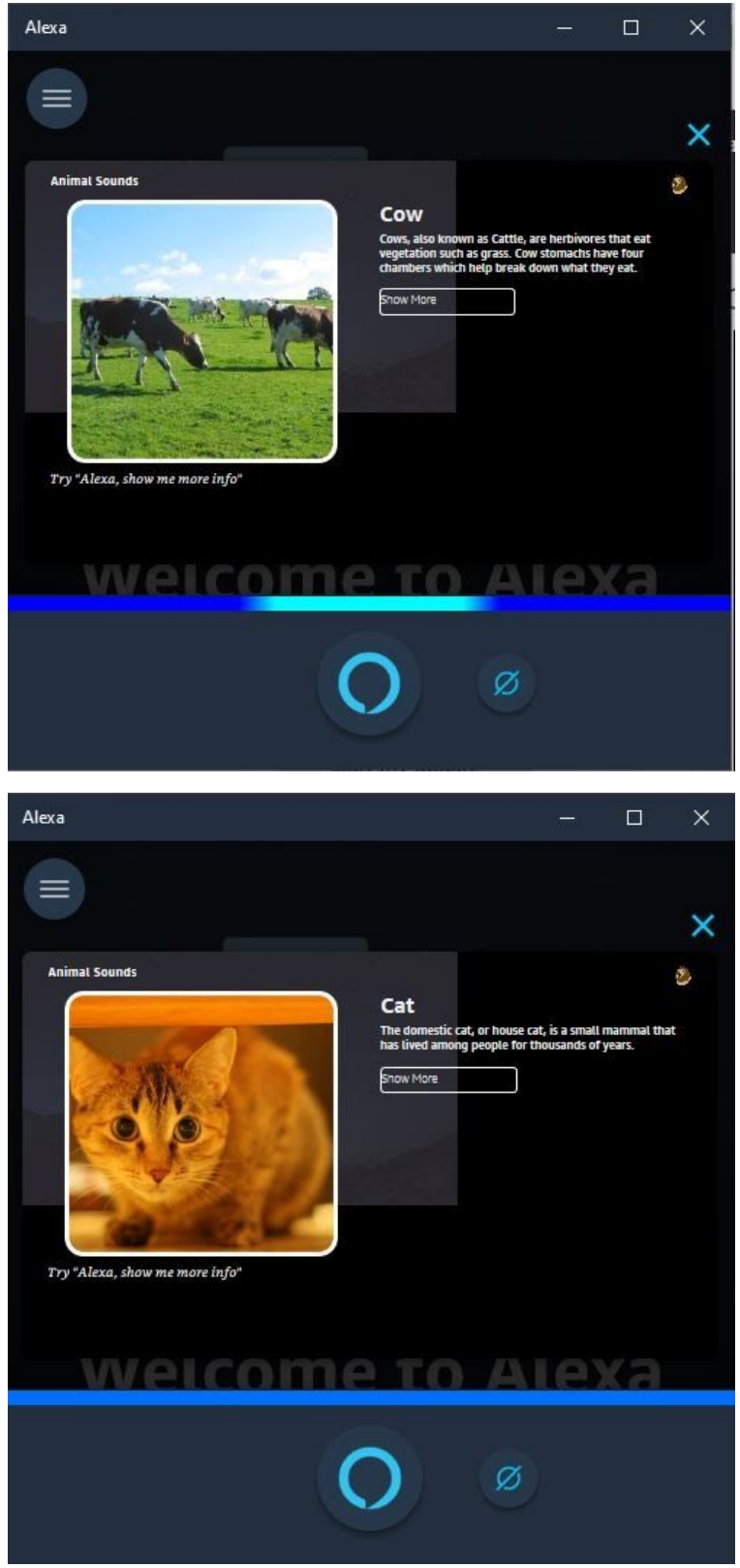

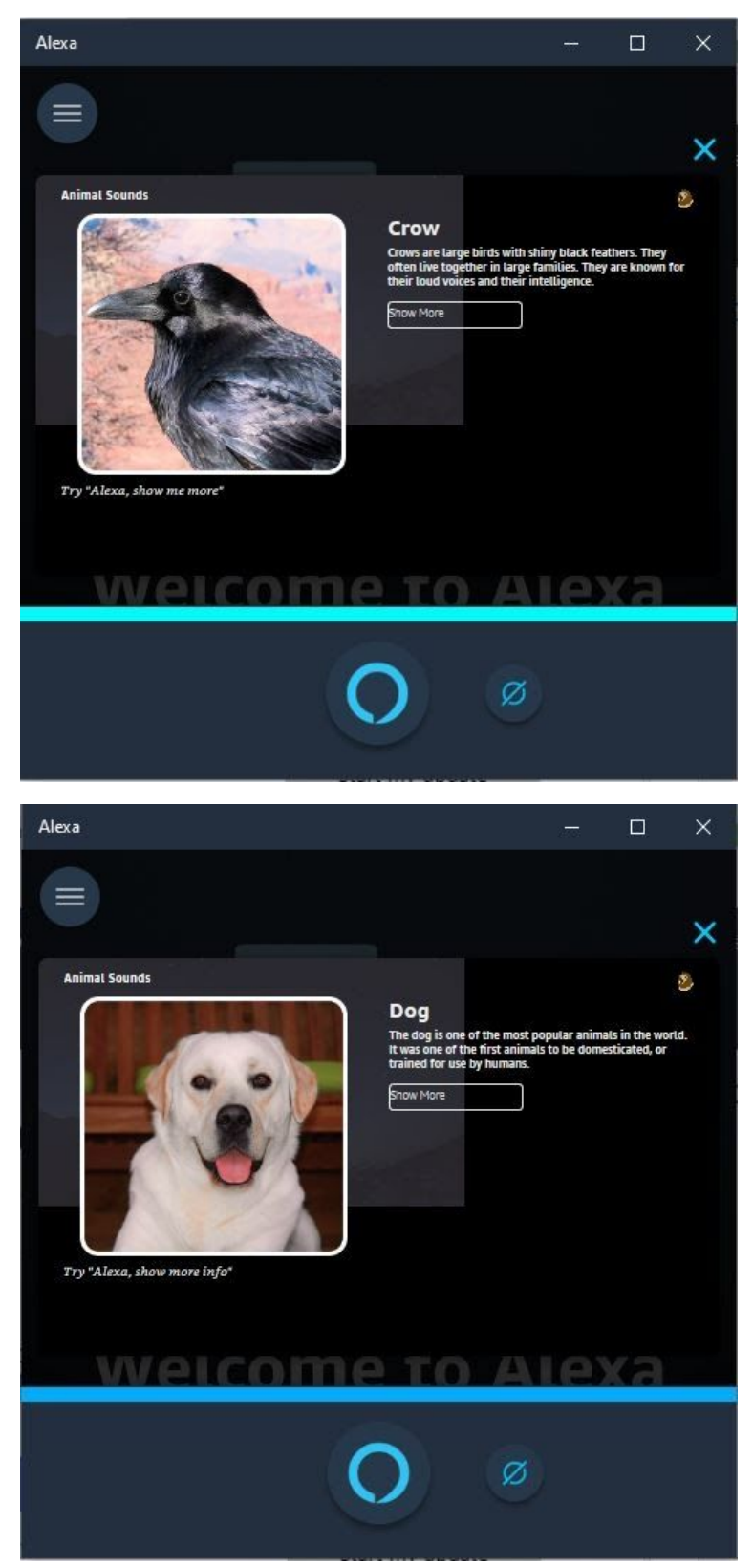

The therapeutic benefits of animal mimicry, closely parallel experiences of stress and trauma in animals, particularly wildlife and a mimicry of their response to stressful, threatening and possible traumatic events and memories. A similar release mechanism is deduced in human mimicry of the supposed natural language of animals as semiotics.(Tønnessen \& Tüür, 2014) argues for the continuum in the animal-human identity, a mechanism for an efficacy of use of animal language semiotics in human natural language based therapy, ranging from self hypnosis techniques to neuro linguistic programming.

\section{Participants.}

Dr Bheemaiah, Anil Kumar, the author, performed 10 sessions ranging from 10 to 15 minutes, using the Alexa Skill 'Animal Sounds.

\section{Materials.}

Amazon Alexa for the Windows 10 PC, was used with the Alexa Skill 'Animal Sounds'.

\section{Design.}

The skill supports several modes:

1. Play an animal sound.

2. Play a game of recognizing an animal sound.

3. List all animal sounds.

The session consists of two parts, requesting Alexa to mimic common animal sounds and repeating in animal mimicry, to achieve a purpose similar to brain spotting with glance. Instead emotion is channeled in animal cries to identify trauma, sentiment in thought and memory, with or without the context of time and space and vitality of an animal and natural connection, supplemented with the naturalness used to annul that trauma.

In the second part, Alexa plays a game of mimicking an animal sound, the subject is asked to mimic the same sound with a 
primordial connection to the animal nature in himself, with an attempt to root out depression, lower stress and eliminate trauma, by a natural connection to one's animal side. This session can last ten to fifteen minutes a day for three weeks.

\section{Procedure.}

A quantitative assessment of the efficacy of animal mimicry therapy as a group therapy in a wilderness setting is part of a future research project in wilderness therapy.

\section{Discussion and Future Work.}

We have thus described several theories supporting, beneficial therapeutic effects of animal mimicry as a form of OBT, therapy. The personal experience of the author proves the effective use of the therapy as a form of wilderness therapy for anxiety, depression, detoxing, and for autism spectrum disorder.

Future work involves a study of the effectiveness of animal mimicry as a group therapy in a wilderness setting with several autism spectrum quantitative scales, like SRS-2, SRS-A and other measures.

\section{References.}

Byström, K., Grahn, P., \& Hägerhäll, C. (2019).

Vitality from Experiences in Nature and Contact with Animals - A Way to Develop Joint Attention and Social Engagement in
Children with Autism? International

Journal of Environmental Research and

Public Health, 16(23).

https://doi.org/10.3390/ijerph16234673

Demille, S. M. (2014). Treatment Outcomes for Struggling Adolescents in an Outdoor Behavioral Health Care Program. In PsycEXTRA Dataset.

https://doi.org/10.1037/e554842014-001

Gass, M. A., "Lee” Gillis, H. L., \& Russell, K. C. (2020). Adventure Therapy: Theory, Research, and Practice. Routledge. https://play.google.com/store/books/details? id=1RPWDwAAQBAJ

[No title]. (n.d.). Retrieved June 30, 2020, from https://www.tandfonline.com/doi/full/10.10 80/0886571X.2013.852452?src=recsys

Russell, K. C., \& Gass, M. A. (2014). Overview of Outdoor Behavioral Health Care Research. In PsycEXTRA Dataset. https://doi.org/10.1037/e555582014-001

Tønnessen, M., \& Tüür, K. (2014). Introduction: The semiotics of animal representations. In The Semiotics of Animal Representations 
(pp. 7-30). unknown.

https://doi.org/10.1163/9789401210720_00

2

Website. (n.d.).

https://doi.org/10.1111/dmcn.13337

White, W. (2015). Stories from the Field: A

History of Wilderness Therapy.

https://books.google.com/books/about/Stori

es_from_the_Field.html?hl=\&id $=\mathrm{eH} 7 \mathrm{djgE}$

ACAAJ 